${ }^{1}$ Centro de Habilidades Clínicas Facultad de Medicina Universidad de Chile. Santiago, Chile.

2Departamento de Medicina Interna Occidente, Facultad de Medicina Universidad de Chile. Santiago, Chile. ${ }^{3}$ Departamento de Fonoaudiología, Facultad de Medicina Universidad de Chile. Santiago, Chile. aFonoaudióloga.

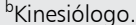

Trabajo no recibió financiamiento. Los autores declaran no tener conflictos de interés.

Recibido el 14 de agosto de 2019, aceptado el 8 de junio de 2020.

Correspondencia a:

Dr. Sergio Bozzo Navarrete Dirección Centro de Habilidades Clínicas, Facultad de Medicina Universidad de Chile.

Av. Las Palmeras 299, Interior Parque Quinta Normal. Santiago, Chile. sbozzo@uchile.c

\section{Descripción y análisis de ECOE con pacientes simulados en internado de Medicina Interna 2016-2017 en Facultad de Medicina Universidad de Chile}

\author{
SERGIO BOZZO NAVARRETE ${ }^{1,2}$, CLAUDIA ARANCIBIA SALVO ${ }^{1,3, a}$, \\ DANIEL CONTRERAS REYES ${ }^{1,2}$, LEONARDO PÉREZ GONZÁLEZ ${ }^{1, \mathrm{~b}}$
}

\section{Structured clinical examinations with simulated patients during internal medicine clerkship}

Background: Practice is essential in clinical training and can be carried out with simulations. The medical school of the University of Chile performs Objective Structured Clinical Examinations (OSCE) using standardized simulated patients for interns. Aim: To report the experience with OSCE in an environment with a high number of students. Material and Methods: Four hundred sixty-two students, participated in three OSCEs during 2016 and 2017, during their internal medicine clerkship. Each OSCE consisted of five scenarios of ambulatory care patients with prevalent medical conditions. Every student had to perform history taking, physical examination and deliver a diagnostic hypothesis, and a plan for management and counseling. Simulated patients provided feedback. To assess validity and reliability, in an argument-based model, planning and activity development were declared. The results were evaluated according to students' and organization variables, internal consistency, interobserver and theoretical concordance tests. Results: No significant bias was found by demographic features or evaluation moment. Internal consistency was good (Cronbach 0.8). Simulated patient evaluators had a high correlation with medical evaluators $(r=0.7)$. There was a low correlation between OSCE results and theoretical tests. Conclusions: OSCE's continuously assess competences during internal medicine clerkship in an environment with a high number of students.

(Rev Med Chile 2020; 148: 810-817)

Key words: Clinical Competence; Education, Medical; Patient Simulation; Simulation Training.

\footnotetext{
L
} a formación de profesionales de la salud depende de la práctica clínica para el logro de las competencias ${ }^{1}$ requeridas en el perfil de egreso de la institución formadora, en respuesta a los requerimientos de la sociedad y en busca de una atención de salud segura y de calidad ${ }^{2-5}$. Esta necesidad formativa se enfrenta a las limitaciones de acceso a pacientes en contextos reales que, entre varias razones, destaca proteger al paciente de los errores propios del proceso formativo de estudiantes $^{6-8}$. Lo anterior, ha impulsado el uso de la simulación en docencia clínica que, además de velar por la seguridad del paciente, brinda oportunidades de práctica seguras, estructuradas y estandarizadas ${ }^{9-11}$.

El trabajo con pacientes simulados/estan- 
darizados (PSE), en particular, permite desarrollar evaluaciones formativas y sumativas en niveles superiores de la escala de Miller ${ }^{12-14}$. Las funciones del PSE se encuentran más allá de la interpretación de roles (casos clínicos, generalmente) e incluyen retroalimentación y evaluación del desempeño de estudiantes ${ }^{15-17}$. Su adecuado cumplimiento requiere de un trabajo dirigido, iterativo y que considere estándares de buenas prácticas $^{18,19}$. Hay experiencias a nivel nacional publicadas sobre el trabajo con PSE, especialmente participando en el Examen Clínico Objetivo Estructurado (ECOE) ${ }^{20-22}$, pero aún dista de lo que se realiza en centros de USA, Europa y $\mathrm{Asia}^{23}$.

El ECOE, conocido como OSCE en inglés $(O b-$ jective Structured Clinical Examination) desde su descripción original ${ }^{24,25}$, ha variado en diseño y se ha extendido por el mundo ${ }^{26,27}$, especialmente en evaluaciones sumativas y de alta relevancia para los evaluados (exámenes de título, certificación de grados académicos, entre otros ${ }^{28}$. En el ECOE, el estudiante muestra sus competencias en múltiples estaciones acotadas y consecutivas, que incluyen atención de PSE, realización de procedimientos y preguntas respecto de situaciones prácticas. Las pautas de evaluación y su aplicación son estandarizadas. Hay publicaciones respecto de experiencias y variantes del ECOE, así como de sus propiedades psicométricas, desarrollo y validez, así como guías para optimizar su uso.

En la Facultad de Medicina de la Universidad de Chile se realizan ECOEs hace más de quince años, incorporando crecientemente la participación de PSE. A partir del año 2011, la formación del Centro de Habilidades Clínicas ( $\mathrm{CHC})$, cuenta con un equipo docente y técnico especializado, además de una Unidad de PSE, y una infraestructura acorde, ha permitido una concentración de recursos. El ECOE del Internado de Medicina Interna (IMI) ha sido modificado reflexivamente para progresar en la entrega de oportunidades de práctica clínica ambulatoria a todos los estudiantes (aproximadamente 240 por año), de carácter evaluativo tanto formativo como sumativo.

Este artículo muestra la factibilidad de su desarrollo continuo en el tiempo, con un número de estudiantes relativamente alto para nuestro medio, con estándares de buenas prácticas y con evidencias de validez y confiabilidad, según modelo basado en argumentos ${ }^{29}$. Este proceso y sus resultados constituyen un desafío que requiere de evaluaciones iterativas, así como de una retroalimentación de los logros alcanzados, y de los puntos que deben atenderse para mejorar.

\section{Material y Método}

Estudio de tipo longitudinal. Se realizaron 3 ECOEs con PSE a todos los estudiantes del IMI, durante los años 2016 y 2017, en el CHC. Los estudiantes firmaron, previamente, contrato de ficción y consentimiento informado. El presente estudio fue aprobado por el Comité de Ética de Investigación en Seres Humanos (CEISH) de la Facultad de Medicina de la Universidad de Chile.

La rotación del IMI dura 16 semanas, se realiza en forma cuatrimestral, de manera que el total de estudiantes rota en tres grupos independientes y sucesivos, durante el sexto año de la carrera de medicina (Figura 1). La práctica asistencial supervisada del internado es realizada en cinco hospitales de alta complejidad de Santiago, a los que son asignados los estudiantes (denominados campos clínicos: centro, norte, occidente, oriente, sur). Se registran los datos de internos/as: edad, género, y centro asistencial de procedencia (Tabla 1).

Cada estudiante participó en tres ECOEs $\left(\mathrm{E}_{0}, \mathrm{E}_{1}\right.$ $\mathrm{y} \mathrm{E}_{2}$ ), sucesivos, mensuales (Figura 1 ). Se requirieron 8 sesiones para cada ECOE y así completar el total de estudiantes. En cada sesión de ECOE, los casos son diferentes y cada interno/a participa en 5 escenarios de atención ambulatoria, con casos clínicos de medicina interna. En cada escenario, el estudiante realizó una atención clínica de un paciente nuevo o control, con historia clínica, examen físico $y$, en algunos casos, exámenes complementarios. Además, debió responder las consultas del paciente y explicar sus hipótesis diagnósticas e indicaciones. Al completar la atención, el estudiante escribió sus hipótesis diagnósticas fundamentadas e indicaciones de manejo (Registro Clínico Electrónico, RCE). Cada escenario comprendió un tiempo de atención de $20 \mathrm{~min}$ y, para RCE, de $5 \mathrm{~min}$. En este modelo de encuentros clínicos, el PSE rota por los boxes de atención. En $E_{0}$ y $E_{1}$, inmediatamente concluido el escenario, el PSE retroalimenta al estudiante en forma verbal y personal, en aspectos de comunicación, de acuerdo con esquema preparado y en base a conductas observadas durante atención clínica. 


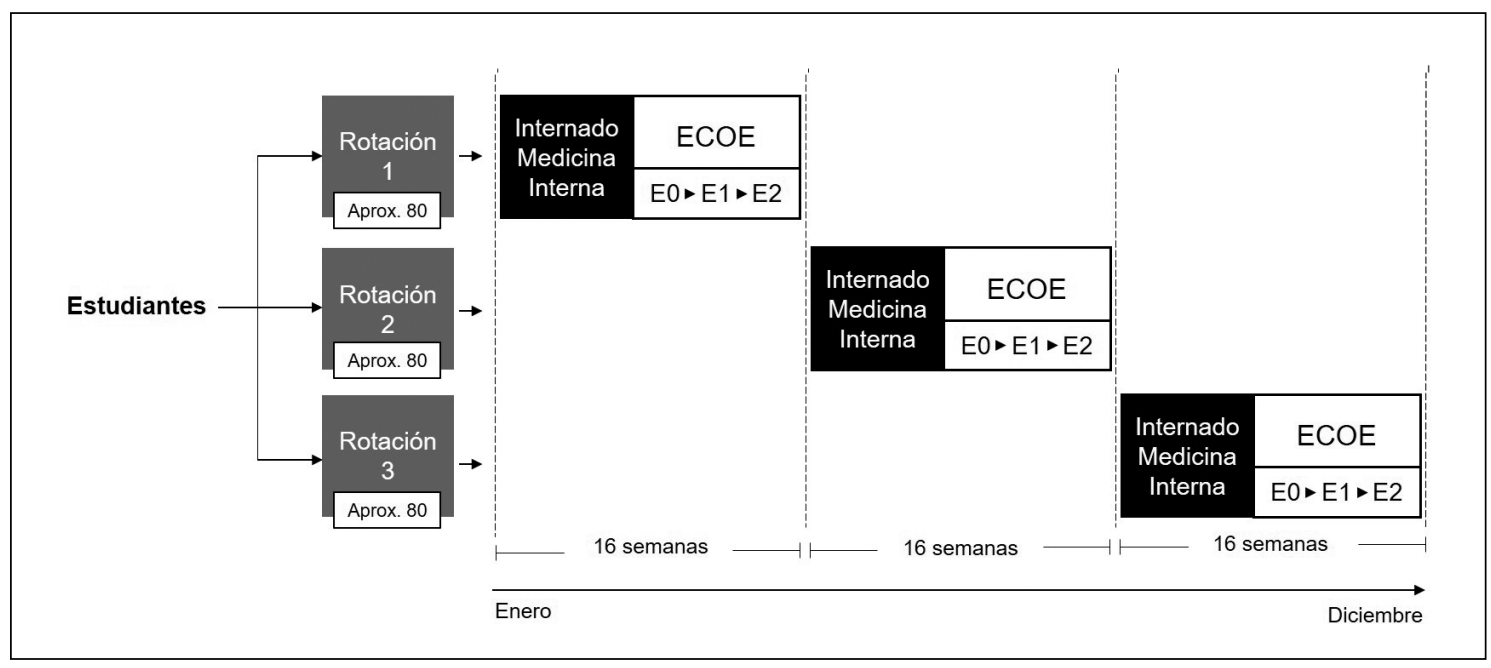

Figura 1. Organización de rotaciones de internado de Medicina Interna y ECOEs. Distribución anual de rotaciones de estudiantes de medicina por Internado de Medicina Interna y realización de ECOEs.

Tabla 1. Caracterización de población en estudio

\begin{tabular}{|c|c|c|c|}
\hline Año & 2016 & 2017 & Global \\
\hline $\mathrm{N}^{\circ}$ internos/as & 241 & 221 & 462 \\
\hline $\begin{array}{l}\text { Género } \\
\qquad \mathrm{M}\left(\mathrm{N}^{\circ} ; \%\right) \\
\mathrm{H}\left(\mathrm{N}^{\circ} ; \%\right)\end{array}$ & $\begin{array}{r}146 ; 60,6 \% \\
95 ; 39,4 \%\end{array}$ & $\begin{array}{r}145 ; 65,6 \% \\
76 ; 34,4 \%\end{array}$ & $\begin{array}{l}291 ; 63 \% \\
171 ; 37 \%\end{array}$ \\
\hline Edad (prom + ds) & $24,4 \pm 1,3$ & $24,2 \pm 1,7$ & $24,3 \pm 1,5$ \\
\hline $\begin{array}{l}\text { Campus práctica asistencial } \\
\text { - Centro } \\
\text { - Occidente } \\
\text { - Oriente } \\
\text { - Norte } \\
\text { - Sur }\end{array}$ & $\begin{array}{l}41 \\
43 \\
45 \\
75 \\
37\end{array}$ & $\begin{array}{l}39 \\
47 \\
37 \\
68 \\
30\end{array}$ & $\begin{array}{r}80 \\
90 \\
82 \\
143 \\
67\end{array}$ \\
\hline
\end{tabular}

La retroalimentación post escenario fue de $5 \mathrm{~min}$. En $\mathrm{E}_{0}$ se enfatizan la inducción y la evaluación formativa de los estudiantes. Al finalizar los tres ECOEs, cada estudiante participó en 15 escenarios con PSE (Tabla 2).

El repositorio de casos y la tabla de especificaciones, que incluye los casos a evaluar, así como las habilidades clínicas a considerar (comunicación, anamnesis y razonamiento) (Tabla 3), fueron preparados en consideración a los requerimientos formativos incluidos en el programa de IMI, por Profesores Encargados de Internado, académicos supervisores y expertos en simulación. Se desarrollaron los casos clínicos con aspectos biográficos y de personalidad del paciente a representar (personaje), así como su motivo de consulta e historia clínica (guion). La Unidad de Paciente Simulado del CHC participó en la elaboración de casos, selección y preparación de los PSE encargados de su representación, evaluación y retroalimentación. Los docentes, además, prepararon pautas de evaluación de conductas observadas ( $\mathrm{PdO})$, en forma de rúbricas politómicas ("realizado", "parcialmente realizado", "no realizado"), de 10 
Tabla 2. Descripción del diseño de ECOEs*

\begin{tabular}{|lccc|}
\hline & ECOE 0 & ECOE 1 & ECOE 2 \\
\hline Casos por interno/a & 5 & 5 & 5 \\
\hline Evaluación & Formativa & Formativa y sumativa & Sumativa \\
\hline Retroalimentación & Sí & Sí & NO \\
Evaluación por escenario & & & \\
- Pauta observación PSE & Sí (PSE, docentes) & Sí (PSE, docentes) & Sí (PSE, docentes) \\
- Respuestas clínicas electrónicas & Sí (docente) & Sí (docente) & Sí (docente) \\
Análisis de resultados & No & Sí & Sí \\
\hline
\end{tabular}

*Cada ECOE se distribuyó en 8 sesiones de 10 a 12 estudiantes.

\section{Tabla 3. Repositorio de casos clínicos* y habilidades clínicas evaluadas, con su ponderación}

\begin{tabular}{|c|c|c|c|}
\hline \multicolumn{2}{|l|}{ Paciente nuevo } & \multicolumn{2}{|l|}{ Control de paciente } \\
\hline \multicolumn{2}{|c|}{ 1. Neumonía adquirida de la comunidad } & \multicolumn{2}{|c|}{$\begin{array}{l}\text { 1. Neumonía adquirida de la comunidad sin respuesta a } \\
\text { tratamiento }\end{array}$} \\
\hline \multicolumn{2}{|c|}{ 2. Neumonía en paciente inmunosuprimido } & \multicolumn{2}{|l|}{ 2. Asma bronquial en crisis } \\
\hline \multicolumn{2}{|l|}{ 3. Asma bronquial atópica } & \multicolumn{2}{|c|}{ 3. Pielonefritis aguda complicada } \\
\hline \multicolumn{2}{|l|}{ 4. Pielonefritis aguda } & \multicolumn{2}{|c|}{ 4. Diabetes mellitus tipo 2 descompensada } \\
\hline \multicolumn{2}{|c|}{ 5. Debut diabetes mellitus tipo 1} & \multicolumn{2}{|c|}{ 5. Hipertensión arterial crónica } \\
\hline \multicolumn{2}{|l|}{ 6. Diabetes mellitus tipo 2} & \multicolumn{2}{|l|}{ 6. Enfermedad renal crónica } \\
\hline \multicolumn{2}{|c|}{ 7. Angina de reciente aparición } & \multicolumn{2}{|c|}{ 7. Enfermedad pulmonar obstructiva crónica } \\
\hline \multicolumn{2}{|l|}{ 8. Osteoartritis } & \multicolumn{2}{|c|}{ 8. Reflujo gastroesofágico complicado } \\
\hline \multicolumn{2}{|l|}{ 9. Lumbago mecánico } & \multicolumn{2}{|l|}{ 9. Úlcera péptica } \\
\hline \multicolumn{2}{|c|}{$\begin{array}{l}\text { 10. Exacerbación de enfermedad pulmonar obstructiva } \\
\text { crónica }\end{array}$} & \multicolumn{2}{|c|}{ 10. Daño hepático crónico no debido a alcohol } \\
\hline \multicolumn{2}{|l|}{ 11. Hemorragia digestiva alta } & \multicolumn{2}{|l|}{ 11. Anemia ferropriva } \\
\hline \multicolumn{2}{|l|}{ 12. Síndrome anémico } & \multicolumn{2}{|l|}{ 12. Hipotiroidismo } \\
\hline \multicolumn{4}{|l|}{ 13. Síndrome colon irritable } \\
\hline \multicolumn{4}{|l|}{ 14. Diarrea crónica } \\
\hline \multicolumn{4}{|l|}{ 15. Síncope } \\
\hline Instrumento & Habilidad clínica & Ponderación & Evaluadores \\
\hline Pauta de observación & $\begin{array}{l}\text { Comunicación } \\
\text { Anamnesis }\end{array}$ & $\begin{array}{l}25 \% \\
25 \%\end{array}$ & $\begin{array}{l}\text { PSE/Docente }{ }^{* *} \\
\text { PSE/Docente*** }\end{array}$ \\
\hline Registro clínico electrónico & $\begin{array}{l}\text { Diagnóstico y sus fundamento } \\
\text { Indicaciones }\end{array}$ & $\begin{array}{l}25 \% \\
25 \%\end{array}$ & $\begin{array}{l}\text { Docentes } \\
\text { Docentes }\end{array}$ \\
\hline
\end{tabular}

* La selección y distribución de casos en cada sesión de ECOE fue aleatoria. **Evaluación simultánea, en una muestra aleatoria, para evaluación de correlación entre observadores.

a 15 ítems según caso, que incluyen aspectos de comunicación y anamnesis. Se entrenó a docentes y PSE para la aplicación de pautas. El total de los estudiantes fue evaluado por los PSE, un porcentaje menor, fue evaluado simultáneamente por do- centes (190 de 2.310 evaluaciones). Se realizaron, también, rúbricas de revisión de los RCE para cada caso, orientadas a evaluar diagnósticos e indicaciones y ser aplicadas por docentes. Los registros de PdO y RCE, son digitales, y se consideraron 
para análisis los porcentajes de logro obtenidos en cada uno de ellos, para cada escenario, en $\mathrm{E}_{1} \mathrm{y}$ $\mathrm{E}_{2}$. Los escenarios fueron registrados en video. $\mathrm{La}$ selección y distribución de casos en cada sesión de ECOE fue aleatoria.

Los resultados de cada estudiante, separando porcentajes obtenidos por caso, se introdujeron en una planilla digital, para su análisis en software SPSS v.24. Se evaluaron medidas de tendencia central, pruebas de normalidad de D'Agostino \& Pearson, y se compararon resultados de rendimiento mediante pruebas pareadas de Wilcoxon (diferencia estadísticamente significativa $\mathrm{p}<0,05$ ). Además, para la consistencia interna de la evaluación (PdO más RCE) se utilizó el Alfa de Cronbach, mientras que, para evaluar la influencia de las variables de internos/as (edad, género, campo de origen) y organización (rotación, sesión) sobre el rendimiento de los estudiantes, se aplicaron modelos de regresión lineal múltiple. A través de la prueba de Spearman, se compararon las evaluaciones de con PdO de PSE y, simultáneamente, docentes (correlación interobservador), con una muestra probabilística (nivel de confianza 95\%, error $6 \%$ ) y, además, se compararon los resultados del ECOE con los de las pruebas de selección múltiple que paralelamente se realizaron durante el internado.

\section{Resultados}

Del total de 481 estudiantes, se incluyeron para análisis los datos de 462 internos/as (96,04\%). La diferencia está dada por quienes no completaron su internado en el período correspondiente y, por tanto, no realizaron el total de ECOEs.

Se dispuso de la totalidad de las evaluaciones de cada interno/a (10 PdO y $10 \mathrm{RCE}$ ), con resultados expresados en términos porcentuales, tanto de E1 como E2, para análisis, separados, con un total de 9.240 registros (4.620 PdO más 4.620 $\mathrm{RCE})$. Se analizan sus resultados por separado y en conjunto, como promedio de $\mathrm{PdO}$ y RCE para cada interno/a.

Se analizó la incidencia de las características de los estudiantes (edad, género y campo clínico de procedencia), así como de su momento de realización del ECOE (rotación de internado y número de sesión), sobre los promedios de porcentajes de logro en $\mathrm{E}_{1}$ y $\mathrm{E}_{2 \text {, }}$ mediante regresión de modelamiento lineal. No se encontró incidencia significativa para las variables edad, género, procedencia y número de sesión, tanto en $\mathrm{E}_{1}$ como $\mathrm{E}_{2}$, en todas las rotaciones. La tercera rotación, correspondiente a los estudiantes que habían realizado dos internados previamente al IMI, fue la única variable que tuvo alguna incidencia positiva significativa, solamente en $\mathrm{E}_{2}$, sobre el porcentaje de logro final.

Para indagar un probable valor formativo del ECOE, se compararon los rendimientos por separado y en conjunto, en PdO y RCE, de $\mathrm{E}_{1}$ a $\mathrm{E}_{2}$, y se encontró un aumento significativo, dado fundamentalmente por mejoría en resultados de RCE (Figura 2).

A su vez, se evaluó la consistencia interna de la evaluación a través del Alfa de Cronbach, considerando conjuntamente los porcentajes obtenidos en PdO más RCE, en $\mathrm{E}_{1}$ y $\mathrm{E}_{2}$. El valor de alfa para el $\mathrm{E}_{1}$ fue de 0,721 mientras que para el $\mathrm{E}_{2}$ fue de 0,785 . Si se consideran ambas evaluaciones, como un proceso de evaluación longitudinal $\left(\mathrm{E}_{1}\right.$ más $\left.\mathrm{E}_{2}\right)$, la fiabilidad sube a 0,801 .

Por otra parte, se compararon los porcentajes de logro según PSE y docentes aplicando PdO

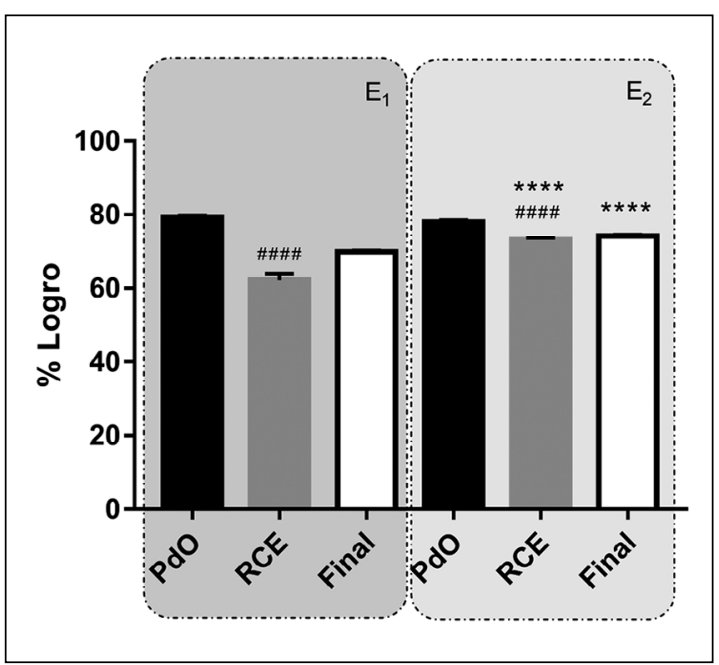

Figura 2. Desempeño en ECOE 1 y 2 desagregado por pauta de observación y registro clínico electrónico. $E_{1}$ y $E_{2}$ corresponden a ECOE 1 y 2 respectivamente, PdO y RCE corresponden a Pauta de Observación y Registro Clínico Electrónico. Se aplicó estadística de comparación de muestras pareadas no paramétricas (wilcoxon, ${ }^{*} \mathrm{o} \# \mathrm{p}<0,05$ ). ${ }^{* * * *}$ corresponde a la comparación de la misma categoría entre $E_{1}$ y $E_{2}$. Mientras que \#\#\#\# corresponde a comparación de PdO y RCE de forma separada en $E_{1}$ y $E_{2}$. 


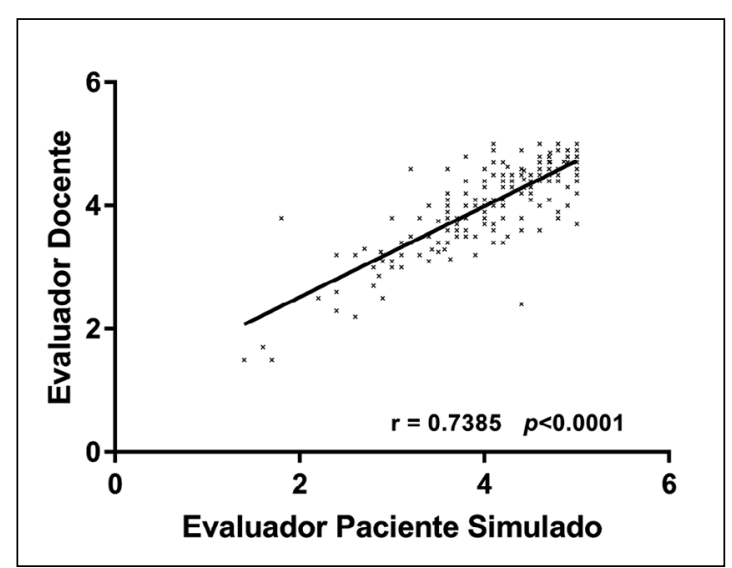

Figura 3. Correlación entre evaluaciones usando Pautas de Observación aplicadas por pacientes simulados y por docentes. Correlación de Spearman entre las Pautas de Observación evaluadas por docentes y pacientes simulados, $n=190$.

durante $\mathrm{E}_{1 .}$ De un total de 2.310 evaluaciones, se dispuso de docentes para 190 evaluaciones simultánea a través de espejo unidireccional, usando la misma PdO. Se encontró una correlación positiva estadísticamente significativa $(r=0,739$; $\mathrm{p}<0,0001$ ) (Figura 3).

Se compararon los resultados obtenidos en las evaluaciones teóricas mediante pruebas con preguntas de selección múltiple (porcentajes de respuestas correctas), con el rendimiento obtenido en los ECOEs (porcentaje promedio PdO más RCE), y se encontró una muy baja correlación $(r=0,2)$.

\section{Discusión}

Los datos recolectados muestran la factibilidad de exponer a cientos de estudiantes a múltiples escenarios de alta complejidad y fidelidad, en rotaciones organizadas en múltiples sesiones. La mayoría de los estudiantes completaron los ECOEs $y$, quienes no lo hicieron, fue por asuntos ajenos a la actividad. La consecución de esto requiere del trabajo de equipos docente, técnico y de PSE, guiados por estándares de buenas prácticas. Es un proceso complejo y costoso, en el que destaca la conformación de la Unidad de PSE, encargada de reclutar, entrenar, organizar y supervisar al equipo de PSE. Además, el desafío de generar casos clínicos, asociados a instrumentos de evaluación, pautas de retroalimentación, suma la necesidad de un equipo docente que, en forma similar a lo que se solicita a estudiantes, mejore progresivamente sus competencias en la formación y evaluación de habilidades clínicas. El equipo técnico es fundamental en la preparación de escenarios, la recolección de datos y la logística que permite organizar las rotaciones sucesivas de estudiantes.

La disponibilidad de una infraestructura acorde a las necesidades de uso es un punto fundamental, lo que fue considerado en la construcción de las actuales dependencias del CHC. Tanto las actividades a realizar como el número de estudiantes a recibir son importantes a considerar antes de la construcción de un centro y adquisición de recursos ${ }^{30}$.

Se destaca que la evaluación del desempeño de los estudiantes es independiente de factores demográficos, de su procedencia de práctica asistencial y, especialmente, del momento en que les toca la actividad. La influencia de la información entregada por quienes han participado en sesiones anteriores, suele ser un punto de discusión que plantea dudas sobre la validez y confiabilidad de la evaluación. Además, hay una muy baja correlación entre los resultados obtenidos en las evaluaciones teóricas, enfocadas en contenidos, respecto a las de los ECOEs, dirigidas a competencias. Planteamos la complementariedad de estos instrumentos como parte del conjunto de metodologías de evaluación empleadas durante el IMI.

Los mejores resultados observados en el desempeño de los estudiantes en la última sesión sugieren un valor formativo, acorde a lo esperado, según el diseño de la experiencia, así como lo señalado por la literatura ${ }^{18-20}$.

Sin duda, el aprendizaje paralelo en las prácticas asistenciales del internado puede influir en mejores resultados, así como cierto "aprendizaje de la metodología". Esto último hace referencia a los aspectos propios de la actividad que influyen en la evaluación del estudiante y que no dependen de sus conocimientos, habilidades (por ejemplo, ansiedad, desconocimiento, desinformación, sesgos, etc.). Para disminuir su impacto, se informa a los estudiantes sobre los pormenores de la actividad y se incluye una jornada completa idéntica, para todos, sin evaluación sumativa. Consentimiento informado y contrato de ficción son útiles en establecer explícitamente los deberes y derechos de los participantes. La incorporación de prácticas con PSE al currículo, así como la difusión de es- 
tándares de actividades, disminuyen la necesidad de inducciones y preparaciones de los estudiantes.

El aporte de la retroalimentación de los PSE puede ser relevante y merece mayor desarrollo e investigación. La correlación interobservadores es aceptable, valida el rol como evaluadores de los PSE y, es un aspecto que puede mejorar.

En términos generales, la automatización del manejo de datos y uso de recursos estadísticos, permitió disponer de información segura y completa.

En relación a validez del ECOE, se ha planteado un enfoque basado en argumentos, con mirada global al proceso $^{29}$, con espacio para declarar sus aspectos constituyentes en un sentido amplio ${ }^{30-32}$. Resulta, a su vez, una guía de planificación, desarrollo y cierre de actividades tipo ECOE. En la experiencia acá expuesta, son argumentos de validez:

- Participación de docentes expertos en contenido para desarrollo de casos auténticos, acorde a programa de internado.

- Diseño de tabla de especificaciones.

- Selección y desarrollo de los instrumentos de evaluación.

- Entrenamiento de evaluadores y PSE.

- Estudiantes deben mostrar competencias en tareas clínicas de la vida real.

- Alfa de Cronbach 0,8.

- Interobservador 0,7.

- Muy baja correlación con evaluaciones teóricas.

Es necesario, seguir desarrollando esta actividad. Considerado lo formativo, se puede avanzar en retroalimentación e incluir debriefing con estudiantes, los que podrían disponer, además, de sus videos y pautas evaluadas. Para el docente sería útil disponer de resultados y seguimientos desagregados por competencias, para planificar remediales y modelar el currículo. Como evaluación sumativa, las exigencias al ECOE deben ajustarse a las implicancias de sus resultados, basando su validez en argumentos, lo que es fundamental en exámenes de título, certificación de grados o recertificación de títulos profesionales.

Como limitaciones, no hubo evaluación de las técnicas de examen físico, dada la necesidad de formación de PSE sobre esta habilidad clínica.

La aleatoriedad de casos a evaluar sin una inducción a los temas específicos de cada caso es discutible y requiere más reflexión.

\section{Referencias}

1. Norman GR. Defining competence: a methodological review. In: Neufeld VR, Norman GR, eds. Assessing Clinical Competence. New York, NY: Springer; 1985; 15-35.

2. Khan K, Ramachandran S Conceptual framework for performance assessment: competency, competence and performance in the context of assessments in healthcare-deciphering the terminology. Med Teach 2012; 34 (11): 920-8.

3. Ten Cate T, Snell L, Carraccio C. Medical competence: The interplay between individual ability and the health care environment. Med Teach 2010; 32: 669-75. doi: 10.3109/0142159X.2010.500897.

4. Dornan T, Littlewood S, Margolis A, Scherpbier A, Spencer J, Ypinazar V. How can experience in clinical and community settings contribute to early medical education? A BEME systematic review. Medical Teacher 2009; 28 (1): 3-18.

5. Carraccio C, Englander R, Van Melle E, Ten Cate O, Lockyer J, Chan MK, et al. Advancing competency-based medical education: a charter for clinician-educators. Acad Med 2016; 91 (5): 645-9.

6. Aggarwal R, Mytton OT, Derbrew M, Hananel D, Heydenburg M, et al. Training and simulation for patient safety. Qual Saf Health Care 2010; suppl 2: 34-43.

7. Ramani S, Leinster S AMEE Guide no. 34: Teaching in the clinical environment. Med Teach 2008; 30 (4): 34764. 10.1080/01421590802061613.

8. Ministerio de Salud Chile. LEY-20.584 Derechos y Deberes de las Personas en Atención de Salud [Internet]. Biblioteca del Congreso Nacional. 2012. Disponible de: http://www.leychile.cl/Navegar?idNorma=1039348.

9. Leaning J. Human rights and medical education: Why every medical student should learn the Universal Declaration of Human Rights. BMJ 1997; 315: 1390.

10. Moya P, Ruz M, Parraguez E, Carreño V, Rodríguez A, Froes P. Efectividad de la simulación en la educación médica desde la perspectiva de seguridad de pacientes. Rev Med Chile 2017; 145 (4): 514-26. https://dx.doi. org/10.4067/S0034-98872017000400012.

11. Boursicot K. Structured assessments of clinical competence. British Journal of Hospital Medicine 2010; 71 (6): 342-4.

12. Wass V, Jones R, van der Vleuten C. Standardized or real patients to test clinical competence? the long case revisited. Med Educ 2001; 35: 321-5.

13. Collins JP, Harden RM AMEE Medical Education Guide No. 13: Real patients, simulated patients and simulators in clinical education. Med Teach 1998; 20: 508-21. 
14. Van der Vleuten C, Sawnson DB. Assessment of clinical skills with standardized patients: state of the art. Teaching Learning Med 1990; 2: 58-76.

15. Barrows HS. An overview of the uses of standardized patients for teaching and evaluating clinical skills: AAMC. Acad Med 1993; 68: 443-51.

16. Gomez JM, Prieto L, Pujol R, Arbizu T, Vilar L, Pi F, Borrel F, Roma J \& Martínez-Carretero JM. Clinical skills assessment with standardized patients. Med Educ 1997; 31: 94-8.

17. Sanson-Fisher RW, Poole AD. Simulated patients and the assessment of medical students' interpersonal skills. Med Educ 1980; 14: 249-53.

18. Lewis K, Bohnert C, Gammon W, Hölzer H, Lyman L, Smith C, Thompson T, Wallace A Gliva-McConvey G. The Association of Standardized Patient Educators (ASPE) Standards of Best Practice (SOBP). Advances in Simulation 2017; 2: 10.

19. Solís I, Bozzo S, Kunakov N. Pacientes estandarizados en la formación de habilidades clínicas, en educación médica de pregrado. Rev Med Chile 2013; 141 (9): 1216-7.

20. Moore P, Leighton M, Alvarado C, Bralic C. Pacientes simulados en la formación de los profesionales de salud: el lado humano de la simulación. Rev Med Chile 2016; 144 (5): 617-25. https://dx.doi.org/10.4067/S003498872016000500010.

21. Morales C, Álvarez N, Candia P, Celedón F, Meyer L, Lombardic, et al. Experiencia con el empleo del examen OSCE en el ciclo de evaluación de competencias clínicas de médicos que revalidan su título. Rev Med Chile 2018; 146 (3): 341-3. https://dx.doi.org/10.4067/s003498872018000300341.

22. Triviño X, Vásquez A, Mena A, López A, Aldunate M,
Varas M, et al. Application of Objective Structured Clinical Examination (OSCE) for pediatric internship assessment in two schools of medicine. Rev Med Chile 2002; 130 (7): 817-24.

23. Patricio MF, Julião M, Fareleira F, Carneiro A. Is the OSCE a feasible tool to assess competencies in undergraduate medical education? Med Teach 2013; 35: 503-14.

24. Harden R, Stevenson M, Downie W, Wilson GM. Assessment of clinical competence using objective structured examination. Br Med J 1975; 1 (5955): 447-51.

25. Harden R. What is an OSCE? Medical teacher 1988; 10 (1): 19-22.

26. Khan KZ, Ramachandran S, Gaunt K, Pushkar P. The objective structured clinical examination (OSCE) AMEE guide no. 81. Part I: an historical and theoretical perspective. Medical teacher 2013; 35 (9): 1437-46.

27. Adamo G. Simulated and standardized patients in OSCEs: achievements and challenges 1992-2003. Med Teach 2003;25(3):262-70.

28. Boulet JR, Smee SM, Dillon GF, Gimpel JR. The use of standardized patient assessments for certification and licensure decisions. Simul Healthc 2009;4(1):35-42.

29. Daniels V, Pugh D. Twelve tips for developing an OSCE that measures what you want. Medical Teacher 2018; 40 (12): 1208-13. DOI: 10.1080/0142159X.2017.1390214.

30. Dent J. Current trends and future implications in the developing role of clinical skills centres. Medical Teacher 2001; 23 (5): 483-9. DOI: 10.1080/01421590120075724.

31. Cook DA, Brydges R, Ginsburg S, Hatala R. A contemporary approach to validity arguments: a practical guide to Kane's framework. Med Educ 2015; 49: 560-75.

32. Kane MT. Validating the interpretations and uses of test scores. J Educ Meas 2013; 50: 1-73. 